\title{
Surgical preperitoneal space: holy plane of dissection between transversalis fascia and preperitoneal fascia for TEPP inguinal hernioplasty
}

\begin{abstract}
Background: Preperitoneal space between peritoneum and transversalis fascia is no longer recognized as a single anatomical entity because presence of a definite thin but membranous 'preperitoneal fascia' which divides the preperitoneal space into two compartments. There is a wide range of surgeons' opinion about anatomical plane of preperitoneal dissection and mesh placement during the laparoscopic inguinal hernioplasty.

Patients and methods: A prospective doctoral research was conducted between 2010 and 2015 under ethical approval and informed consent. Laparoscopic total extraperitoneal preperitoneal (TEPP) hernioplasty was done in 60 adult patients with inguinal hernias (unilateral, 52; bilateral, 8) through posterior rectus approach with 3-midline-port technique.

Results: Interfascial dissection between transversalis fascia and preperitoneal fascia was found avascular and straightforward, and this plane was readily extendable not only around cord structures into inguinal canal but also across midline to contralateral side. Mesh placement in this interfascial preperitoneal plane was associated with minimal intra-operative and post-operative complications. Prefascial (anterior to transversalis fascia) and retrofascial/true preperitoneal (posterior to preperitoneal fascia) dissections were tedious and associated with significant intra-operative complications.

Conclusion: Interfascial preperitoneal dissection between transversalis fascia and preperitoneal fascia was avascular, readily extendable to inguinal canal and opposite side. Keeping the fascia/fat on both sides of this 'surgical preperitoneal plane' during interfascial dissection was extremely useful and is strongly recommended for laparoscopic inguinal hernioplasty.
\end{abstract}

Keywords: preperitoneal space, holy plane, transversalis fascia, preperitoneal fascia, laparoscopic anatomy, TEP anatomy

\author{
Volume 6 Issue I - 2018
}

\author{
Maulana MAnsari \\ Department of Surgery, Aligarh Muslim University, India
}

Correspondence: Maulana Mohammed Ansari, Ex-Professor of General Surgery, Department of Surgery, JN Medical College, Aligarh Muslim University, UP, India, Tel 009| 95574492 I2, Email mmansari.amu@gmail.com

Received: February 14, 2018| Published: March 05, 2018
Abbreviations: TEPP/TEP, total extraperitoneal preperitoneal hernioplasty; TAPP, trans-abdominal preperitoneal hernioplasty; PRS, posterior rectus sheath

\section{Introduction}

“...traditional hernia topics are not stale or outmoded but, in the light of new observations, are fresh, dynamic and practical in our everyday approach to the problem of hernias". ${ }^{1}$ Preperitoneal/extraperitoneal space between the peritoneum and the transversalis fascia is no longer recognized as a single anatomical entity because of the presence of a definite thin but membranous 'preperitoneal fascia' which is often mistaken as the 'posterior lamina' of the transversalis fascia. ${ }^{2-10}$ However, the preperitoneal fascia clearly divides the preperitoneal space into two compartments, ${ }^{11-14}$ but 'this layer remained unknown to surgeons unfamiliar with older literature'. ${ }^{14}$ 'Presently the laparoscopic totally extraperitoneal inguinal hernioplasty (TEPP/ TEP) has become an established technique for repair of inguinal hernia'. ${ }^{15}$ 'However, there remains significant confusion regarding the transversalis fascia and the multilayered preperitoneal fascia'. There is a wide range of surgeons' opinion about anatomical plane of preperitoneal dissection and mesh placement during the laparoscopic inguinal hernioplasty through either the total extraperitoneal preperitoneal (TEPP) or the transabdominal (TAPP) approach, ${ }^{12,13,16}$ namely, true preperitoneal (retrofascial), interfascial between the transversalis fascia and the preperitoneal fascia/urogenital fascia, and prefascial/ retromuscular anterior to the posterior rectus sheath fascia and transversalis fascia. 'Initially, the dissection of the extraperitoneal space in the TEP approach tended to be difficult, confusing and therefore hard to learn'. ${ }^{17}$ Therefore, we conducted a laparoscopic study for the doctoral thesis for award of $\mathrm{PhD}$ (Surgery), and the observations regarding the avascular preperitoneal plane, the holy plane of dissection during TEPP hernioplasty are presented here.

\section{Material and methods}

A prospective study was designed and conducted in the elective clinical settings in the Jawaharlal Nehru Medical College Hospital, Aligarh w.e.f. April, 2010 to November, 2015, under the approval of the Institutional Ethics Committee and the Committee for Advanced Scientific Research of Aligarh Muslim University, Aligarh, India. Adult patients with inguinal hernia were recruited for the laparoscopically 
total extraperitoneal preperitoneal (TEPP) hernioplasty under the written informed consent. All patients were operated by a single experienced senior surgeon, the author. Recruitment Criteria were (1) Patient's choice of the laparoscopic hernia repair, (2) Financial affordability of patients, (3) Patient's fitness for general anaesthesia as judged in the pre-anaesthetic check-up clinic, (4) Availability of functioning laparoscopic equipment and instruments. Inclusion Criteria included (1) adult patient $\geq 18$ years, (2) patients with uncomplicated fully reducible primary inguinal hernia, (3) patients with no co-morbidity or controlled mild co-morbidity (ASA grade I-II only of American Society of Anesthesiologists), and (4) written informed consent for the laparoscopic hernioplasty. Exclusion Criteria were: (1) refusal for laparoscopic repair, (2) patient $<18$ years, (3) patients with uncontrolled or severe co-morbidity (ASA grade IIIIV), (3) complicated/ recurrent inguinal hernia, (5) patient with only femoral hernia, and (6) patient with previous lower abdominal surgery. Patients' demographic characteristics (age, gender, weight (without footwear), height, and occupation) were carefully documented. Patients' body mass index (BMI) was calculated by the Deurenberg's formula of body weight in $\mathrm{Kg}$ divided by square of height in meters. ${ }^{18}$ Posterior rectus sheath approach with 3-midline-port technique with direct telescopic initial dissection without a balloon dissector was utilized under $\mathrm{CO}_{2}$ insufflation pressure of $12 \mathrm{mmHg}$. This was followed by further definitive sharp/blunt dissection by the instruments. Details of the surgical technique were consistently the same as reported earlier by the author. ${ }^{10,19-21}$

\section{Results}

Sixty six patients with inguinal hernia (Male 63; Female 3) were recruited for the study. The three female patients were excluded due to one or more exclusion criteria. Three male patients were excluded due to an early conversion (TAPP 1; Open surgery 2). Cause of the early conversion included (1) frank pneumoperitoneum just after placement of the 1st optical port, (2) instrument injury to the deep inferior epigastric vessels just before placement of the second working port, and (3) patient's haemodynamic instability due to excessive $\mathrm{CO} 2$ retention soon after the start of the procedure. Thus only 68 TEPP hernioplasties were successfully performed in the 60 patients (Unilateral TEPP 52; Bilateral TEPP 8). Mean age of the patients was $50.1 \pm 17.2$ years (range 18-80), and their mean BMI was $22.6 \pm 2.0$ $\mathrm{kg} / \mathrm{m} 2$ (range 19.3-31.2). By profession, the patients were farmers $(\mathrm{N}=6)$, field workers (6), students $(\mathrm{N}=7)$, office workers $(\mathrm{N}=8)$, retired persons $(\mathrm{N}=9)$, and manual labourers $(\mathrm{N}=24)$.

In the first three cases of the study, an indigenously made balloon made of the surgical glove fingerstall, was used to create the preperitoneal space but it was found grossly unsatisfactory for several reasons. Firstly, balloon dissection tore the multi-laminated fascias of abdomino-inguinal and pelvic regions haphazardly; secondly, haphazard tearing of the various fascial layers made it difficult to recognize what is what; thirdly, tearing of the tiny vessels resulted in the some definite oozing which was minor and usually insignificant but coloured the tissues, making difficult the differentiation of the various tissues and tissue planes ; fourthly, in one case, plane was created anterior to the transversalis fascia and the deep inferior epigastric vessels which were brought down on to the floor of the operative field, appearing as a cable running vertically and forming the so-called 'cord sign', and which had to be coagulated and divided to proceed further. After the balloon dissection, an avascular plane of dissection was a matter of trial and error as well as difficult to find and to maintain, resulting in a tedious frustrating scenario. Therefore, telescopic dissection under direct $\mathrm{CO}_{2}$ insufflation after placement of the first blunt $11-\mathrm{mm}$ trocar was utilized instead of the balloon dissection in the remaining part of the study.

The space between the posterior rectus sheath (PRS) and the often well-formed posterior epimysium (rectusial fascia) of rectus abdominis muscle was easily opened up under direct vision with the to-and-fro movement of the $0^{\circ} 10-\mathrm{mm}$ telescope in order to reach the requisite preperitoneal space, because there was minimal loose areolar connections between the infraumbilical posterior rectus sheath and the rectusial fascia (Figures 1A-D). ${ }^{22}$ However, we happened to enter the direct retromuscular plane anterior to the rectusial fascia in two of our cases, and this prefascial plane of dissection anterior to the rectusial fascia was not straightforward with criss-cross vessels present between the rectus abdominis muscle and its posterior epimysium/ rectusial fascia, (Figures 1A1-B2) and a rent was created in this fascia to enter the true posterior rectus canal for further dissection (Figures 1C1-D1). The entry into the preperitoneal space was usually made by opening the transversalis fascia below the arcuate line of the incomplete posterior rectus sheath $(n=54)$; however, in presence of the complete posterior rectus sheath $(\mathrm{n}=14)$ (Figures 1A-D), the posterior rectus sheath itself was first opened to reach the transversalis fascia which was then in turn divided to enter the preperitoneal space behind the transversalis fascia.

In first three cases of the study, the definitive dissection in the true preperitoneal plane between the preperitoneal fascia and the parietal peritoneum was tried but was found tedious due to not only frequent tearing of the bared thin parietal peritoneum, resulting in pneumoperitoneum with further limitation of an already small closed working space but also frequent tearing of the thin preperitoneal fascia and the loose ill-formed extraperitoneal fat, resulting in too frequent loss of the dissection plane. Moreover, working in the true preperitoneal plane was also associated with minor oozing because of tearing of the criss-cross tiny blood vessels supplying both the parietal peritoneum and the preperitoneal fascia/fat. Dissection in the true preperitoneal was therefore abandoned in the further part of the study.

In two cases of the study, direct extension of the initial dissection plane from within the posterior rectus canal inferiorly into the pelvis or laterally into the inguinal region used to lead into the prefascial dissection anterior to the transversalis fascia (Figure 2). It was found associated with two phenomena of surgical significance. Firstly, despite careful dissection, the operative field become sort of bloody due to minor oozing from tearing of the tiny vessels because of the shared vascular supply between the transversalis fascia and the respective musculature, especially the transversus abdominis in the inguinal region; oozing, howsoever minor, was found problematic because of tissue discoloration with difficult appreciation of the different tissue planes and poor visibility in a limited space secondary to significant absorption of the light by the blood. Secondly, the prefascial dissection used to bring down the deep inferior epigastric vessels along with the transversalis fascia down on the floor, which used to interfere with further lateral dissection by their hanging like a cable across the operative field, producing the 'cord sign'. In these cases, the transversalis fascia was therefore entered at a convenient place at about the level of the middle port, and the deep inferior 
epigastric vessels were either hooked up by a trans-cutaneous suture $(n=1)$ or divided after electro-coagulation to facilitate further dissection $(\mathrm{n}=1)$.
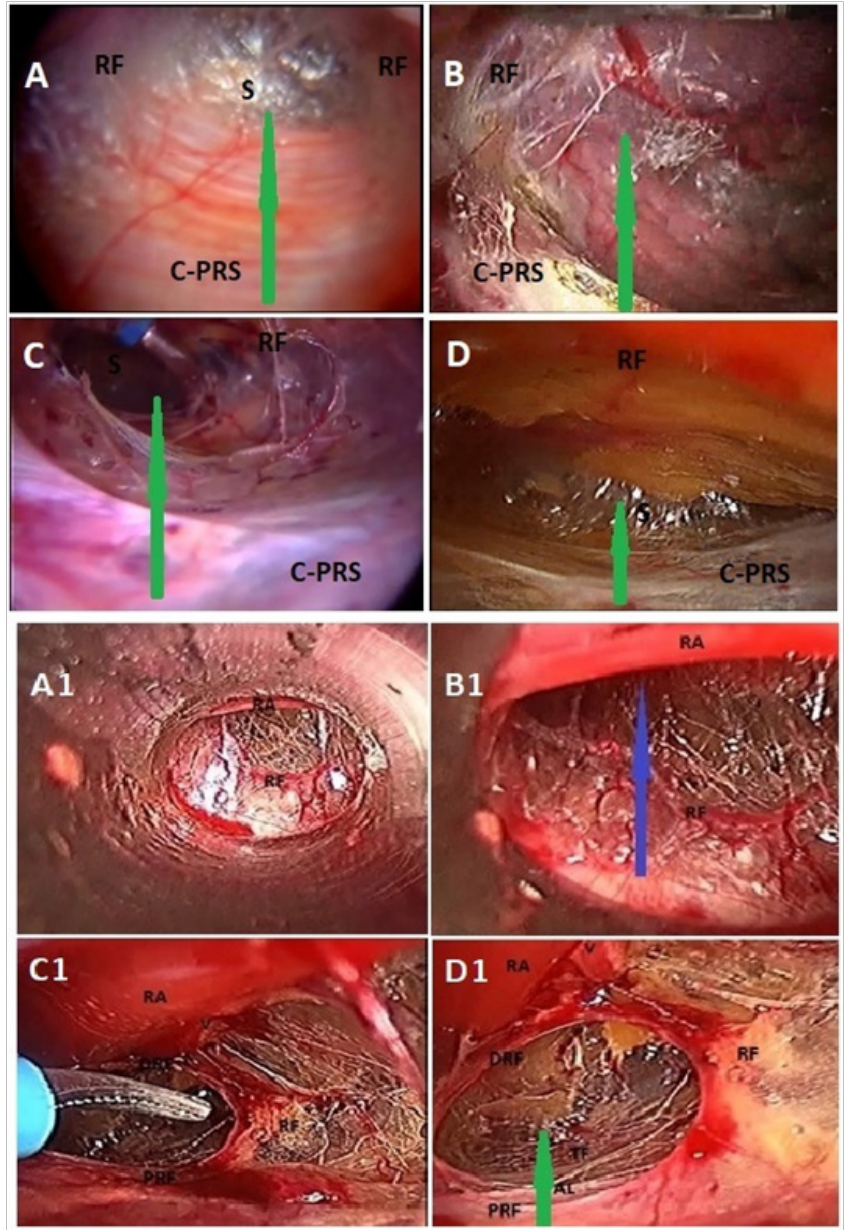

Figure I Total extraperitoneal preperitoneal (TEP) hernioplasty: A-D, shows various morphology of rectusial fascia $(\mathrm{RF})$ and posterior rectus sheath; $\mathrm{A}$, thick membranous rectusial fascia (RF) covering the rectus abdominis muscle (not visible) and aponeurotic posterior rectus sheath (PRS); B, thin membranous rectusial fascia (RF) covering the rectus abdominis muscle (partly visible) and partly aponeurotic posterior rectus sheath (PRS); C, thin flimsy rectusial fascia (RF) covering the rectus abdominis muscle (partly visible) and membranous complete posterior rectus sheath (PRS); D, thick diaphanous rectusial fascia (RF) covering the rectus abdominis muscle (not visible) and grossly attenuated posterior rectus sheath (PRS); $\mathrm{Al}$ and $\mathrm{BI}$, Prefascial dissection anterior to the rectusial fascia (RF); Blue arrow, indicates prefascial plane of dissection $\mathrm{RA}$, rectus abdominis muscle; $\mathrm{V}$, deep inferior epigastric vessels covered by rectusial fascia; $\mathrm{Cl}$ and $\mathrm{DI}$, rectusial fascia opened up to enter the true posterior rectus canal (green arrow); PRF, proximal part of rectusial fascia covering posterior rectus sheath; DRF, distal part of rectusial fascia now covering the rectus abdominis muscle (RA); AL, arcuate line, the lower end of posterior rectus sheath; Green arrow, indicates plane of dissection in true posterior rectus canal; RA, rectus abdominis muscle; (Reproduced with permission from Ansari's Thesis). ${ }^{22}$

In 63 cases of our study, an easily fissile plane of surgical dissection for the definitive space creation was found underneath the transversalis fascia and the deep inferior epigastric vessels which were kept anteriorly abutting the anterior abdominal wall but anterior to the preperitoneal fascia/fat covering the parietal peritoneum (Figure
3). This easily fissile plane was avascular due to presence of only minimal loose areolar tissue between these two distinct fascias, i.e., the transversalis fascia and the preperitoneal fascia, and was invariably found to continue into the deep inguinal ring, thereby making the opportunity to pull the intra-canalicular part (lying within the inguinal canal) of the spermatic cord within the abdomen safely and then to dissect the indirect hernial sac $(\mathrm{n}=60)$ off the cord structures (Figures $3 \mathrm{C}-3 \mathrm{~F})$. In all cases with the direct hernia $(\mathrm{n}=8)$, the hernial sac was easily pulled in and pushed down along with the preperitoneal fascia/ fat by simple blunt dissection underneath the transversalis fascia. After reduction of the direct hernial sac along with the preperitoneal fascia/fat down into the pelvis, the transversalis was always found left in situ anteriorly abutting the abdominal wall musculature, forming the pseudo-sac of the direct inguinal hernia (Figure 3A).
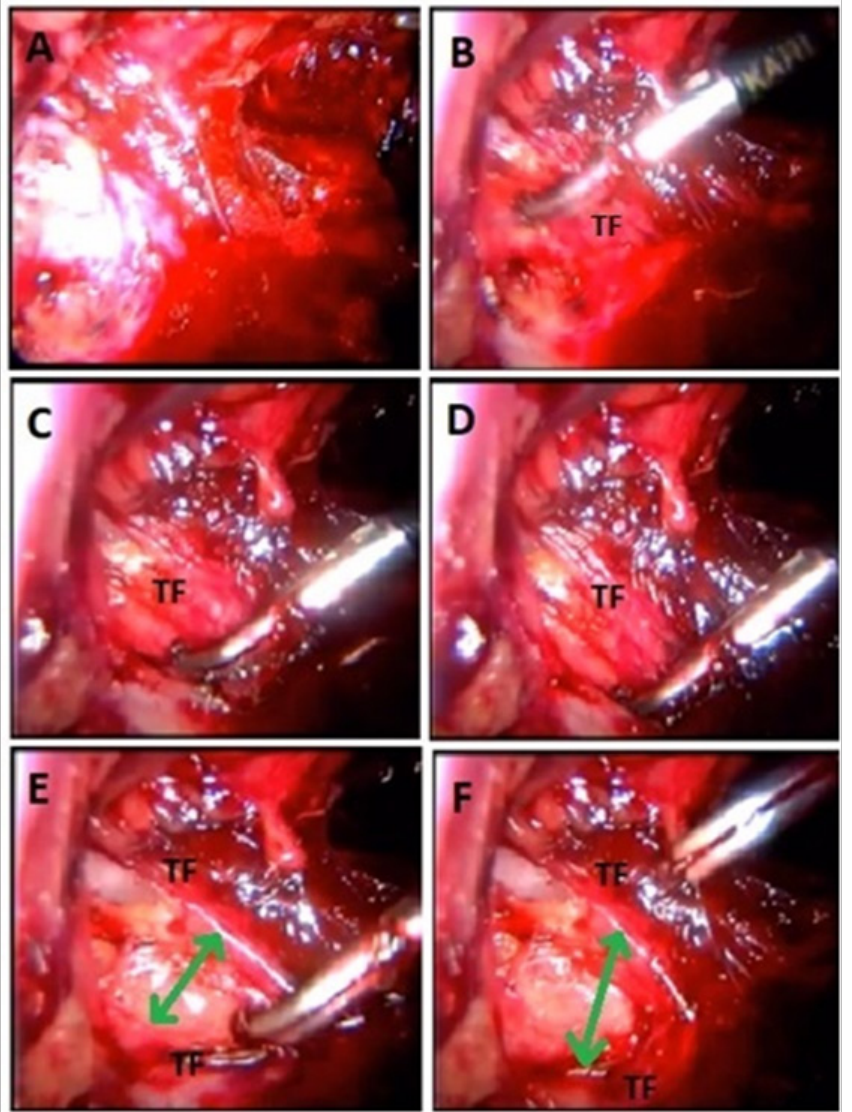

Figure 2 Total extraperitoneal preperitoneal (TEP) hernioplasty: Prefascial dissection anterior to the transversalis fascia (RF) in another patient with grossly attenuated complete posterior rectus sheath initially mistaken for the transversalis fascia; $\mathrm{A}$ and $\mathrm{B}$, indicates bloody dissection anterior to the transversalis fascia; $\mathrm{E}$ and $\mathrm{F}$, shows creation of a rent (double-headed arrow) in the transversalis fascia to enter the surgical preperitoneal space between transversalis fascia and preperitoneal fascia; (Reproduced with permission from Ansari's Thesis). ${ }^{22}$

The easily fissile avascular plane between the transversalis fascia and the preperitoneal fascia was found readily extendable laterally upto the anterior superior iliac spine, the lateral limit of laparoscopic dissection, and medially upto the midline. In 5 out of 8 cases with the bilateral inguinal hernia, the avascular pane between the transversalis fascia and the preperitoneal fascia was easily extendable across the 
midline, making the technical feasibility for successful simultaneous bilateral TEPP hernioplasty. The remaining 3 cases of the bilateral inguinal hernia underwent the interval TEPP hernioplasty on the contralateral side by reason of the longer operating time on the ipsilateral side $(n=2)$ and by the patient's preference $(n=1)$.
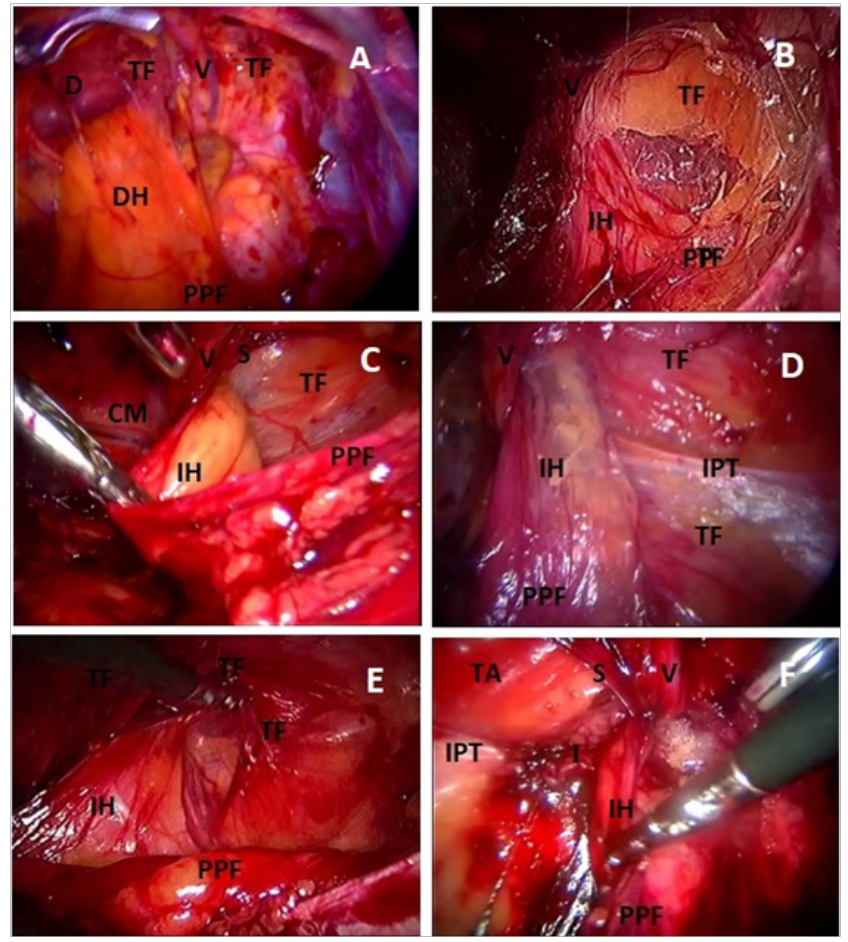

Figure 3 Total extraperitoneal preperitoneal (TEP) hernioplasty: Avascular interfascial dissection between transversalis fascia (TF) and preperitoneal fascia (PPF) in six different patients (A to F); $D$, direct hernial defect; $D H$, direct hernia covered by preperitoneal fascia/fat; $\mathrm{IH}$, indirect hernia ensheathed along with cord structures by preperitoneal fascia; $\mathrm{V}$, deep inferior epigastric vessels covered by transversalis fascia; CM, corona mortis; IPT, iliopubic tract TA, transversus abdominis coming very low in the floor of inguinal canal and covered by a thin transversalis fascia; $\mathrm{S}$, superior sling of transversalis fascia; I, inferior sling of transversalis fascia; (Reproduced with permission from Ansari's Thesis). ${ }^{22}$

While working in the plane between the transversalis fascia anteriorly and the preperitoneal fascia posteriorly, the parietal peritoneum or its extension, the hernial sac, was not seen at all or seen only faintly depending upon the morphology of the preperitoneal fascia/fat (Figures 3B-F). Such an anatomic disposition was consistently observed in all of our patients who underwent TEPP hernioplasty $(\mathrm{N}=68)$. The parietal peritoneum was recognizable or visible only during two phases of further dissection, viz., firstly, when the preperitoneal fascia forming the internal spermatic fascia is entered in the pulled-in intra-canalicular part of the spermatic cord for dissection of the indirect hernial sac either, and secondly, when the preperitoneal fascia which used to ensheathe the cord structures was teased out during parietalization of the cord structures. Peritoneum forming the direct hernial sac was never seen as the intact preperitoneal fascia/fat was also pulled down along with the direct sac (Figure 3A).

In all cases studied, while working within the preperitoneal space between the transversalis fascia and the preperitoneal fascia, the DIEV vessels were found contained and covered by the transversalis fascia below the arcuate line of the incomplete posterior rectus sheath $(n=54)$ but always visible across it clearly or faintly (Figure 3), depending upon the character of the transversalis fascia which was found as a single diaphanous layer $(n=41)$, single membranous layer $(n=10)$ or thin flimsy layer $(n=17)$; however in presence of a complete posterior rectus sheath, the DIEV vessels were found contained and covered by the rectusial fascia while running on the posterior surface of the rectus abdominis muscle (Figures 1-D1). On the other hand, the cord structures and the indirect hernial sac if present, were found enclosed within a distinct fascial layer (internal spermatic fascia) not derived from the transversalis fascia but derived from the preperitoneal fascia (Figure 3 ) which was found as a single membranous layer $(n=56)$ or double membranous layer $(\mathrm{n}=12)$.

\section{Discussion}

Seamless inguinal hernioplasty through open or laparoscopic technique warrants highest degree of combination of accurate anatomic knowledge and astute surgical skill. ${ }^{23-31}$ Moreover, contrary to the open surgery, the laparoscopic hernioplasty puts a much larger burden of laparoscopic experience of about 20-80 cases for developing adequate anatomic understanding and surgical skill to independently perform the repair with precision, rapidity and safety. ${ }^{32-37}$ Folscher et al. ${ }^{12}$ rightly stated that 'The fascial anatomy in the groin is a complex arrangement between peritoneum, urogenital fascia and transversalis fascia'. Dissection between the correct fascial layers allows rapid identification of the exact anatomy in an avascular plane. This ensures true parietalization of the cord structures in an ordered and anatomically correct fashion' ${ }^{12}$ Stoppa's principles of the open giant preperitoneal mesh repair for the inguinal hernias include the identification of a bloodless plane in the interparieto-peritoneal space, and the parietalization of the cord structures. ${ }^{38}$ Based on these principles, the preperitoneal dissection and mesh placement is also carried out during the laparoscopic preperitoneal repair for the inguinal hernia. ${ }^{12}$

In the present study, the prefascial plane of dissection anterior to the transversalis fascia or the retrofascial dissection posterior to the preperitoneal fascia resulted in a bloody field due to tearing of the shared vascular supply between the fascia and its adjacent structure, viz., between the transversalis fascia and the transversus abdominis muscle or between the preperitoneal fascia and the parietal peritoneum. These dissection planes (prefascial and retrofascial) were found unsuitable for the space creation and mesh placement. The plane of surgical dissection was found avascular and easily fissile between the diaphanous transversalis fascia anteriorly and the preperitoneal fascia covering the preperitoneal fat posteriorly, i.e., in other words, this plane of dissection was avascular as long as both fascia \& fat was visible on either side of the dissection plane. Avascular interfascial plane of surgical dissection and mesh placement is illustrated well in the (Figure 4) Our observations confirmed the 'loose fissile plane' of the Australian Paediatric surgeon Dr. Robert Fowler, existing between the transversalis fascia and the preperitoneal fascia, and his guidance criteria of the deep inferior epigastric vessels which 'serve as a guide to the recognition and separation of the preperitoneal fascia from the transversalis fascia' (which contain the vessels). ${ }^{4}$ Redman ${ }^{6}$ and Mirilas et al..$^{13}$ also supported the Fowler's guide ${ }^{4}$ of the deep inferior epigastric vessels for separation of these two distinct fasciae. Such an anatomic dissection is readily explained by their differential embryological origin. In 1950, Mark Hayes ${ }^{39}$ described the development of the 
transversalis fascia from the 'outermost' mesenchymal layer which also participates in the development of the abdominal wall musculature, and the development of the preperitoneal fascia from the 'intermediate' mesenchymal layer which also contributes to the development of the extraperitoneal/preperitoneal fat. The parietal peritoneum is closely applied to the extraperitoneal fibrofatty tissues and mainly develops from the 'innermost' mesenchymal layer. ${ }^{39}$

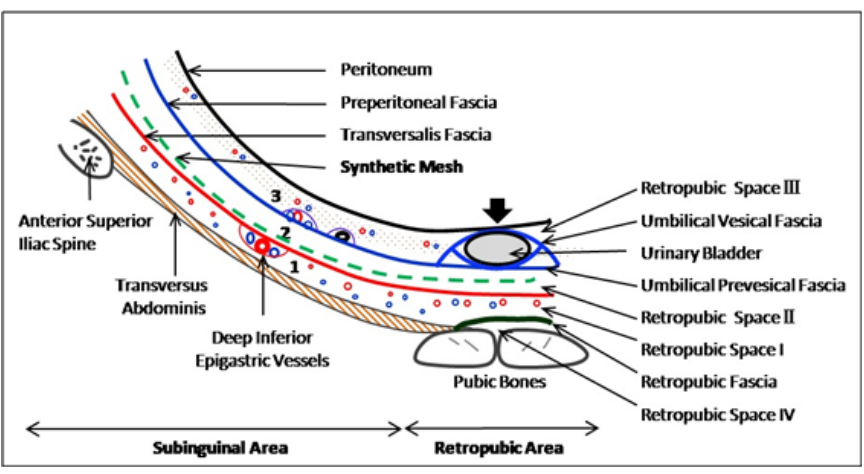

Figure 4 Total extraperitoneal preperitoneal (TEP) hernioplasty: Avascular interfascial preperitoneal dissection and mesh placement (green dashed line) between transversalis fascia (red line) and preperitoneal fascia (blue line) in inguinal region, and between transversalis fascia (red line) and umbilical prevesical fascia (blue line), a localised condensation of the preperitoneal fascia (blue line); I, prefascial space anterior to transversalis fascia; 2, interfascial or surgical preperitoneal space between transversalis fascia and preperitoneal fascia; 3, retrofascial or true preperitoneal space posterior to preperitoneal fascia; Arrow, indicates the area where peritoneum and preperitoneal fascia are firmly adherent to posterior wall of urinary bladder; (Reproduced with permission from Ansari's Thesis. ${ }^{22}$

Sadler, ${ }^{40}$ Hinman $^{41}$ and Redman ${ }^{6}$ further refined the concept of three mesenchymal layers developing into the three well-defined fascial layers in the inguino-pelvic region: inner stratum, the supportive connective tissue of the peritoneum and the visceral fascia; intermediate stratum, the bilaminar extraperitoneal fascia (fatty layer and membranous stroma); and outer stratum, the transversalis fascia and other investing fasciae of the flat muscles of the anterior abdominal wall, and the muscles themselves. Mechanism of development of these fasciae and muscles are recently documented well in the exhaustive work of Mirilas and associates. ${ }^{13}$ Therefore, the present study confirmed that the easily cleavable interfascial plane between the transversalis fascia and the preperitoneal fascia (or the so-called posterior lamina of the transversalis fascia) represent the true Bogros space and not the true preperitoneal space between the preperitoneal fascia and the parietal peritoneum. It was also proved in the present study that the easily fissile interfascial plane (the surgical preperitoneal space or the true Bogros space) of one side could readily be extended to the contralateral side across the surgical preperitoneal space of the retropubic region as suggested earlier by the author. ${ }^{42,43}$ Our findings confirmed the observations of Hureau. ${ }^{44}$

True Bogros space canal and traditional Retzius space are, in reality, not in direct continuity, and communication between them warrants iatrogenic division of the transversalis fascia which forms the posterior boundary of the traditional retropubic space of Retzius and the posterior rectus canal (lower part) generally but which forms the anterior boundary of the true subinguinal space of Bogros. ${ }^{43,45}$ It is because of this reason that the lateral dissection in the inguinal region is often advocated and performed in the true preperitoneal plane (posterior to preperitoneal fascia) but the medial dissection in the retropubic regions is always done in between the transversalis fascia anteriorly and the urinary bladder which lies posteriorly in the urogenital fascia, a part of the preperitoneal fascia, i.e., the medial dissection is always interfascial in nature. ${ }^{13,46}$ Thus the preperitoneal fascia/fat is divided knowingly or unknowingly somewhere to join the lateral and medial dissections in the enthusiasm of the space creation. This common scenario is illustrated well by the detailed descriptions of Folscher et al. ${ }^{12}$ although these authors strongly advocated only true preperitoneal dissection. True preperitoneal plane is never avascular because of the shared blood supply of the preperitoneal fascia/fat and the subjacent peritoneum and its supporting connective tissues. It is possibly this disparate dissection in the lateral and medial areas that Rene Stoppa did not think that the current laparoscopic hernioplasty is really a true representation of his giant preperitoneal prosthetic repair of inguinal hernia. True preperitoneal dissection in the retropubic region is difficult and tedious due to firm adhesion of the parietal peritoneum to the supero-posterior part of the urinary bladder and presence of its closely applied two fasciae (umbilical vesical and prevesical fascias, a localised part of the preperitoneal fascia), and hence fraught with the peritoneal button-holing and the risk of bladder injury with their attendant consequences. ${ }^{9,46}$ The inexperienced surgeon, in order to be true to the book, follows the true preperitoneal plane for the medial dissection (medial to the medial umbilical ligament) similar to the lateral dissection and often lands up in these complications, especially injury to the urinary bladder which is contained within the urogenital part of the preperitoneal fascia. ${ }^{46}$ Therefore the operating surgeon should atleast heed the hybrid concept of Mirilas et al. ${ }^{13}$ to create the space posterior to the preperitoneal fascia in the lateral inguinal region but to go anterior to the preperitoneal fascia (urogenital fascia here) in the medial retropubic region. We created the preperitoneal space for mesh placement anterior to the preperitoneal fascia in both the retropubic and inguinal regions and found this interfascial plane (between preperitoneal fascia and transversalis fascia) straightforward, easily cleavable and quite safe as long as gentle dissection is maintained to a minimum without exposing the nerves in the 'triangle of pain'; we encountered one instance of transient orchalgia possibly due to inadvertent excessive dissection and injury to the genital branch of the genitofemoral nerve, and it is of interest to note that this happened in the 43rd patient in the consecutive order of our TEPP hernioplasties $(\mathrm{N}=68)$, indicating that every laxity in the laparoscopic anatomic dissection always has a price even for the experienced surgeon. However, apprehension of Mirilas and associates ${ }^{13}$ for a higher incidence of inguinodynia secondary to mesh placement in the interfascial plane of the parietal compartment was proved ill-founded in our study.

Understanding the inguino-pelvic anatomy appears difficult because of the presence of multiple fascial layers and their anatomic variations. The various fascial layers may include, from anterior to posterior, the rectusial fascia, ${ }^{19}$ the long incomplete posterior rectus sheath,,$^{20,21,47}$ the complete posterior rectus sheath/fascia which may itself be multilayered, , 7,9,20,21,48-53 the transversalis fascia which is invariably unilaminar $5,10,13,48,53$ but which is often believed to be bilaminar ${ }^{23,25,54-57}$ and the preperitoneal fascia which may itself be multilayered. ${ }^{6,7,9,10}$ Presence of these multiple fascial layers leads to the complexity of the inguinal anatomy with significant confusion still persisting despite the recent laparoscopic literature on the musculoaponeurotic, vascular, and nervous structures of the inguinal area from a transabdominal or preperitoneal vantage point. ${ }^{9,16}$ It is because of this multilayered 
fascial anatomy that Initially, the dissection of the extraperitoneal space in the TEP approach tended to be difficult, confusing and therefore hard to learn' ${ }^{17}$ The steep long learning curve secondary to the complex inguinal anatomy is often found discouraging due to the tedious and often frustrating preperitoneal dissection to many surgeons who therefore disregard the laparoscopic technique for such a basic problem of inguinal hernia. ${ }^{17,58}$ The main cause of difficulties in reality lies in the inadequate understanding of extra-peritoneal fascial anatomy \& improper dissection, ${ }^{7,29,30,59}$ leading to its lack of popularity despite the obvious advantages and better results of the laparoscopic inguinal hernioplasty. ${ }^{16,58}$

The avascular holy plane of the interfascial dissection, better called as the 'Surgical Preperitoneal Plane' remains the same even for those who still believe in the Cooperian concept of the bilaminar transversalis fascia as long as they keep dissecting in between its two layers. ${ }^{16}$ The so-called 'posterior lamina' of the transversalis fascia is in reality the preperitoneal fascia ${ }^{3-10,13,25}$ and 'the concept of the bilaminar fascia transversalis is outdated, ${ }^{54}$

\section{Conclusion}

The preperitoneal surgical dissection was avascular and straightforward as long as both fascia \& fat was visible on both sides of the dissection plane-anteriorly the diaphanous transversalis fascia and posteriorly the preperitoneal fascia covering the preperitoneal fat, and this loose easily fissile avascular plane between the transversalis fascia and preperitoneal fascia was labelled as the surgical preperitoneal space' to differentiate it from the true preperitoneal space. The requisite holy plane of 'surgical preperitoneal space' conformed fully to the Bogros concept of the preperitoneal space. In addition to the technical learning and hands-on training, it is binding on the part of surgeon to acquire accurate flawless knowledge of the complex inguinal multifascial anatomy before performing the technically demanding laparoscopic hernioplasty, ${ }^{29,32,36,37,60-62}$ because the seamless preperitoneal repair of inguinal repair needs mastery of the preperitoneal anatomy to satisfy the Stoppa's basic two principles of preperitoneal repair, namely, timely identification of a bloodless plane in the interparieto-peritoneal space, and adequate parietalization of the cord structures. ${ }^{38}$

\section{Acknowledgements}

All figures and graphs of this article are reproduced with permission from 'Ansari MM. A Study of Laparoscopic Surgical Anatomy of Infraumbilical Posterior Rectus Sheath, Fascia Transversalis \& Pre-Peritoneal Fat/Fascia during TEPP Mesh Hernioplasty For Inguinal Hernia., Doctoral Thesis for PhD (Surgery), Aligarh Muslim University, Aligarh, India, 2016.'

\section{Conflict of interest}

Authors declare there is no Conflict of interest towards this manuscript.

\section{References}

1. Nyhus LM, Klein MS, Rogers FB. Inguinal Hernia. Curr Probl Surg. 1991;28(6):407-450

2. Anson BJ, Morgan EH, McVay CB. Surgical anatomy of the inguinal region based upon a study of 500 body halves. Surg Gynecol Obstet. $1960 ; 111: 707-725$.
3. Lampe EW. Special comment: Experiences with preperitoneal hernioplasty. In: Nyhus LM, Condon RE, editors. Hernia. 1st ed, JB Philadelphia: Lippincott; 1964:295-301.

4. Fowler R. The applied surgical anatomy of the peritoneal fascia of the groin and the "secondary" internal inguinal ring. Aust N Z J Surg. 1975;45(1):8-14.

5. Condon RE. The anatomy of the inguinal region and its relation to groin hernia. In: Nyhus LM, Condon RE, editors. Hernia, 4th ed, Philadelphia: Lippincott; 1995:16-72.

6. Redman JF. The secondary internal ring: Applications to the surgery of the inguinal canal. Clin Urol. 1996;155(1):170-173.

7. Arregui ME. Surgical anatomy of the pre-peritoneal fasciae and posterior transversalis fasciae in the inguinal region. Hernia. 191(2):101-110.

8. Memon MA, Quin TH, Cahill DR. Transversalis fascia: Historical aspects and its place in contemporary inguinal herniorrhaphy. $J$ Laparoendosc Adv Surg Tech. 1999;9(3):267-272.

9. Spitz JD, Arregui ME. Fascial anatomy of the inguinal region. In Robert Bendavid, Jack Abrahamson, editors. Abdominal Wall. Hernias: Principles and Management, 1st ed. (Reprint), Chapter 8, New York: Springer Science-Business Media; 2001: 86-91.

10. Ansari MM. Transversalis Fascia and Preperitoneal Fascia: A Laparoscopic Study of Live Surgical Anatomy during TEPP Hernioplasty - Final Report and Literature Review. Ann Int Med Den Res. 2017;3(6):19-32.

11. Diarra B, Stoppa R, Verhaeghe P, et al. About prolongations of the urogenital fascia into the pelvis: An anatomic study and general remarks on the interparietal-peritoneal fasciae. Hernia. 1997;1(4):191-196.

12. Folscher DJ, Leroy J, Jamali FR. Totally extrafascial endoscopic preperitoneal hernia repair: a merger of anatomy and surgery. The exact description to endoscopically dissect the spermatic fascia. Hernia. 2000;4(4):223-227.

13. Mirilas P, Mentessedou A, SKandalais JE. Secondary internal inguinal ring and associated surgical planes: surgical anatomy, embryology, applications. J Am Coll Surg. 2008;206(3):561-570.

14. Amid PK, Hiatt JR. Surgical anatomy of the preperitoneal space. J. Am Coll Surg. 2008;207(2):295.

15. Lau H, Patil NG, Yuen WK. Day-case endoscopic totally extraperitoneal inguinal hernioplasty versus open Lichtenstein hernioplasty for unilateral primary inguinal hernia in males: a randomized trial. Surg Endosc. 2006;20(1):76-81.

16. Lange JF, Rooijens PPGM, Koppert S, et al. The preperitoneal tissue dilemma in totally extraperitoneal (TEP) laparoscopic hernia repair. Surg Endosc. 2002;16(6):927-930.

17. Felix EL. Laparoscopic Inguinal Hernia Repair. In: Nathaniel J Soper, Lee L Swanstrom, editors. Mastery of Endoscopic and Laparoscopic Surgery, 3rd ed. Chapter 53, Philadelphia: Lippincott Williams \& Wilkins; 2009:523-537.

18. Deurenberg P, Weststrate JA, Seidell JC. Body mass index as a measure of fatness: age- and sex-specific prediction formulas. Br J Nutr. 65(2):105-114

19. Ansari MM. Rectusial Fascia: A New Entity of Laparoscopic Live Surgical Anatomy. Open Access J of Surg. 2017;3(4):1-5.

20. Ansari MM. Posterior Rectus Sheath: A Prospective Study of Laparoscopic Live Surgical Anatomy during TEPP Hernioplasty. World J Lap Surg. 2018;11(1):1-13. 
21. Ansari MM. Posterior rectus sheath variations: surgical significance and clinical implications for laparoscopic hernia surgeons. Int Surg $J$ 2018;5(2):683-694.

22. Ansari MM. A study of laparoscopic surgical anatomy of infraumbilica posterior rectus sheath, fascia transversalis and pre-peritoneal fat/fascia during TEPP mesh hernioplasty for inguinal hernia. Doctoral Thesis for PhD (Surgery), Aligarh Muslim University, India; 2016.

23. Cooper AP. The Anatomy and Surgical Treatment of Abdominal Hernia London: Longman and Co; 1804.

24. Cleland J, Mackay JY, Young BJ. The relations of the aponeurosis of the transversalis and internal oblique muscles to the deep epigastric artery and to the inguinal canal. In: Memoirs and memoranda in anatomy, London: Williams \& Norgate; 1889:142-145.

25. Read RC. Cooper's posterior lamina of transversalis fascia. Surg Gynecol Obstet. 1992;174(5):426-434.

26. Annibali R, Fitzgibbons RJ, Quinn T. Surgical Anatomy of the Inguinal Region from Laparoscopic Perspective. In: Robert Bendavid, Jack Abrahamson, editors. Abdominal Wall Hernias: Principles and Management, Chapter 7, New York: Spinger-Verlag; 2001; 72-85.

27. Spaw AT, Ennis BW, Spaw LP. Laparoscopic hernia repair: the anatomic basis. J Laparoendosc Surg. 1991;1(5):269-277.

28. Rosser J. The anatomical basis for laparoscopic hernia repair revisited. Surg Laparosc Endosc. 1994;4(1):36-44.

29. Leim MS, van Steensel CJ, Boelhouwer RU, et al. The learning curve for totally extraperitoneal laparoscopic inguinal hernia repair. $\mathrm{Am} \mathrm{J}$ Surg. 1996;171(2): 281-285.

30. Faure JP, Doucet C, Rigourd PH, et al. Anatomical pitfalls in the technique for total extra peritoneal laparoscopic repair for inguinal hernias. Surg Radiol Anat. 2006;28(5):486-493.

31. Bhatia P. Understanding Endoscopic Anatomy of Inguinal Region. In Suresh Deshpande, Abhay Dalvi, editors. Comprehensive Laparoscopic Surgery (IAGES Book), 2nd ed, Chapter 13, Kolhapur: Rajhuns Printing Press; 2012:97-111.

32. Edwards CC, Bailey WR. Laparoscopic Hernia Repair: The Learning curve. Surg Laparosc Endosc Percutan Tech. 2000;10(3):149-153.

33. Feliu-Pala X, Martin-Gomez M, Morales-Conde S, et al. The impact of the surgeon's experience on the results of laparoscopic hernia repair. Surg Endosc. 2001;15(12):1467-1470.

34. Lau L, Patil NG, W K Yuen, et al. Learning curve for unilateral endoscopic totally extraperitoneal (TEP) inguinal hernioplasty. Surg Endosc Interv Techn. 2002;16(12):1724-1728.

35. Choi YY, Kim Z, Hur KY. Learning curve for laparoscopic totally extraperitoneal repair of inguinal hernia. Can J Surg. 2012;55(1):3336 .

36. Gupta A, Ashish, Bhandari V, Kumar L, et al. A Run on the Path of Learning Curve of Laparoscopic Hernia Repair (TEP). Sch J App Med Sci. 2015;3(5A):1821-1825.

37. Suguita FY, Essu FF, Oliveira LT, et al. Learning curve takes 65 repetitions of totally extraperitoneal laparoscopy on inguinal hernias for reduction of operating time and complications. Surg Endosc. 2017;31(10):3939-3945.

38. Stoppa R, Petit J, Abourachid H. Procede original de plasties des hernies de l'aine: L'interposition sans fixation d'une prothese en tulle de dacron par voie mediane sous-peritoneale. Chirurgie. 1973;119:321-326.
39. Hayes MA. Abdominopelvic Fascia. Am J Anat 1950; 87(1):119-161. DOI: 10.1002/aja.1000870105

40. Sadler TW. Lagman's medical embryology. 5th ed. Baltimore: Williams and Wilkins: 1985:148-149.

41. Hinman F. Atlas of urosurgical anatomy. Philadelphia: WB Saunders Co; 1993:130.

42. Ansari MM. Retzius Space: Not A Single Anatomical Entity: New Insights, Simplified \& Illustrated in A Laparoscopic Study during TEPP Hernioplasty for Inguinal Hernia. Ann Int Med Den Res. 2017;4(1):SG63-73.

43. Ansari MM. Retzius and Bogros Spaces: A Prospective Laparoscopic Study and Current Perspectives. Ann Int Med Den Res. 2017;3(5):SG2531 .

44. Hureau, J. The space of Bogros and the interparietoperitoneal spaces In: Robert Bendavid, Jack Abrahamson, Maurice E. editors. Abdominal Wall Hernias: Principles and Management, Chapter 11, New York: Spinger-Verlag; 2001; 101-106.

45. Colborn GL, Skandalakis JE. Laparoscopic inguinal anatomy. Hernia. 1998;2(4):179-191.

46. Arregui ME, Navarrete J, Davis CJ, et al. Laparoscopic inguinal herniorrhaphy: Techniques and controversies. Surg Clin North Am. 1993;73(3):513-527.

47. Monkhouse WS, Khalique A. Variations in the composition of the human rectus sheath: a study of the anterior abdominal wall. $J$ Anat. 1986;145: 61-66.

48. McVay CB, Anson BJ. Composition of the rectus sheath. Anat Rec. 1940;77(2):213-225.

49. McVay CB. The anatomic basis for inguinal and femoral hernioplasty. Surg Gynecol Obstet. 1974;139:931-945.

50. Askar OM. Surgical anatomy of the aponeurotic expansions of the anterior abdominal wall. Ann R Coll Surg Eng. 1977;59:313-321.

51. Moffat DB. Lecture Notes on Anatomy, Oxford: Blackwell Scientific Publications; 1987:201-209.

52. Rizk NN. The arcuate line of the rectus sheath-does it exist? J Anat. $1991 ; 175: 1-6$

53. Mwachaka PM, Saidi HS, Odula PQ, et al. Locating the arcuate line of Douglas: is it of surgical relevance. Clin Anat. 2010;23(1):84-86.

54. Mirilas P. Intertransversalis approach for laparoscopic urology: surgical anatomy concerns. Arch Surg. 2012;147(10):980.

55. Morton T. The Surgical Anatomy of Inguinal Herniae, the Testis and Its Coverings. London, UK: Taylor \& Walton, 1941.

56. Mackay JY. The relations of the aponeurosis of the transversalis and internal oblique muscles to the deep epigastric artery and to the inguinal canal. In: Cleland J, editor. Memoirs and Memoranda in Anatomy. London: Williams \& Norgate; 1889:143-146.

57. Lytle WJ. Internal inguinal ring. Br J Surg. 1945;32:441-446.

58. Swanstrom LL. Comment on 'Chapter: Laparoscopic Inguinal Hernia Repair'. In: Nathaniel J Soper, Lee L Swanstrom, editors. Mastery of Endoscopic and Laparoscopic Surgery. 3rd ed. Chapter 53, Philadelphia: Lippincott Williams\&Wilkins; 2009:537.

59. Spitz JD, Arregui ME. Sutureless laparoscopic extraperitoneal inguinal herniorrhaphy using reusable instruments: Two hundred three repairs without recurrence. Surg Laparosc Endosc. 2000;10:24-29. 
60. Arregui MR, Davis CD, Yucel O, et al. Laparoscopic mesh repair of inguinal hernia using a preperitoneal approach: a preliminary report. Surg Laparosc Endosc. 1992;2:53-58.

61. DeTurris SV, Cacchione RN, Mungara A, et al. Laparoscopic herniorrhaphy beyond the learning curve. J Am Coll Surg. 2002;194:6573 .
62. Schouten N, Elshof JWM, Simmermacher RKJ, et al. Selecting patients during the "learning curve" of endoscopic Totally Extraperitoneal (TEP) hernia repair. Hernia. 2012;17(6):737-743. 